\title{
Descriptions of four new dextral land snails of the genus Camaena (Gastropoda, Eupulmonata, Camaenidae) from south China
}

\author{
Pei Wang', Mei-Ling Hu', Jun-Hong Lin ${ }^{1,2}$, \\ Hai-Fang Yang ${ }^{3}$, Xiao-Jing Li', Wei-Chuan Zhou'
}

I Key Laboratory of Molluscan Quarantine and Identification of GACC, Fuzhou Customs District, Fujian 350001, China 2 College of Plant Protection, Fujian Agriculture and Forestry University, Fuzhou, Fujian 350002, China 3 National Wetland Museum of China, Hangzhou, Zhejiang, 310013, China

Corresponding author: Wei-Chuan Zhou (wczhou@163.com); Mei-Ling Hu (93770092@qq.cn)

Academic editor: M.Schilthuizen | Received 12 May 2020 | Accepted 6 November 2020 | Published 24 November 2020

http://zoobank.org/CF98F8DE-F863-419E-B904-183D85779CAB

Citation: Wang P, Hu M-L, Lin J-H, Yang H-F, Li X-J, Zhou W-C (2020) Descriptions of four new dextral land snails of the genus Camaena (Gastropoda, Eupulmonata, Camaenidae) from south China. ZooKeys 996: 37-58. https://doi. org/10.3897/zookeys.996.54187

\begin{abstract}
In this study, four new dextral camaenid from China are reported, based on shell morphology, reproductive system anatomy, and molecular phylogenetic analyses: Camaena funingensis Zhou, Wang \& Lin, sp. nov., Camaena gaolongensis Zhou, Wang \& Lin, sp. nov., Camaena maguanensis Zhou, Wang \& Hu, sp. nov., and Camaena yulinensis Zhou, Wang \& Hu, sp. nov. Detailed descriptions of the morphological characteristics including shells and genitalia, DNA sequences, and living environments of the four new species are provided, with further comparisons with congeners.
\end{abstract}

\section{Keywords}

Anatomy, Camaena, molecular biology, shell morphology, terrestrial snail

\section{Introduction}

The genus Camaena was established by Albers (1850). It is the speciose type genus in the family Camaenidae, with the type species Helix cicatricosa Müller, 1774. The species in this genus are mainly distributed throughout southern China, Indochina, and

Copyright PeiWang et al. This is an open access article distributed under the terms of the Creative Commons Attribution License (CC BY 4.0), which permits unrestricted use, distribution, and reproduction in any medium, provided the original author and source are credited. 
beyond in Southeast Asia, and most are locally endemic (Pilsbry 1894; Zilch 19591960, 1964; Richardson 1985; Chen and Gao 1987; Ding et al. 2016; Inkhavilay et al. 2019). The genus was divided into five subgenera (Camaena Albers, 1850, Camaenella Pilsbry, 1893, Pseudobba Moellendorff, 1891, Pancala Kuroda \& Habe, 1949, Miyakoia Minato, 1980) on the basis of classifications by Pilsbry (1894), Kuroda and Habe (1949), Zilch (1959-1960), and Vaught (1989). A recent molecular phylogeny (Hoso et al. 2010) and anatomical study (Hwang 2012) suggested that Pancala and Miyakoia should be synonyms of the confamilial genus Satsuma.

There are 24 species of the genus distributed in southern China belonging to two subgenera, Camaena and Camaenella. Twenty-three species belong to Camaena (Yen 1939; Chen and Zhang 1999; Schileyko 2003; Ai et al. 2016; Ding et al. 2016), and only one species is in Camaenella (Pilsbry 1894; Yen 1939; Chen and Zhang 1999). The subgenus Camaenella was treated as a synonym of Camaena or as a genus in its own right by some scholars (Chen and Gao 1987; Chen and Zhang 1999). In this article, Camaenella will be considered as a valid subgenus.

Camaena species are divided into a sinistral group and a dextral one. They are usually characterized by a moderately solid shell with scar-like protrusions or malleations, 4.5-5.5 slightly convex whorls, a brown or yellow surface with red or puce spiral bands, and reflexed aperture margins (Schileyko 2003; Ai et al. 2016). The classification of Camaena has mainly relied on the shell features. Anatomical and molecular studies of Camaena are rare, except for the sinistral and the newly described species (Chen and Zhang 1999; Ai et al. 2016; Ding et al. 2016; Páll-Gergely et al. 2016; Wu et al. 2019). Historically, the classification of this genus is rather confused. For the sinistral group, the taxonomic status has always been controversial, and scientific names have been revised repeatedly. Ding et al. (2016) revised C. cicatricosa as four species, C. cicatricosa, C. inflata (Möllendorff, 1885), C. obtecta (Fischer, 1898), and C. connectens (Dautzenberg \& Fischer, 1906), and described one new species C. poyuensis Zhou, Wang \& Ding, 2016 using morphological and molecular studies. In the same year, Ai et al. (2016) described two new species C. lingyunensis Zhou \& Lin, 2016 and C. detianensis Zhou \& Lin, 2016 according to shell morphology, reproductive system and molecular biology. Thus, the sinistral Camaena group contains 12 species or subspecies to date (Schileyko 2003; Ai et al. 2016; Ding et al. 2016). The dextral group can be divided into three informal subgeneric groups according to the morphological characteristics of the shell, especially the shape and location of the carina.

1. Group I possesses an acute and moderate carina on the body whorl. This group could be further divided into two categories by shell height i.e., a relatively low and flat spire, which includes C. longsonensis (Morlet, 1891), C. jinpingensis Chen, Zhang \& Li, 1990 and C. vorvonga (Bavay \& Dautzenberg, 1900); a relatively high spire, e.g., C. vayssierei (Bavay \& Dautzenberg, 1909).

2. Group II possesses a blunt carina, which is placed on the higher or middle parts of the body whorl, such as C. vulpis (Gredler, 1887), C. leonhardti (Möllendorff, 1888), and C. choboensis (Mabille, 1889). 
3. Group III possesses a smooth periphery, e.g., C. hainanensis (Adams, 1870) and C. xanthoderma (Möllendorff, 1882).

In this study, the authors have examined many specimens collected in Guangxi and Yunnan in southern China between 2013 and 2015, and discovered four new dextral species on the basis of morphological, anatomical, and molecular evidence, and living environments.

\section{Materials and methods}

Specimens were collected by the authors from several sites in China (Fig. 1). The longitude and latitude were recorded using a GPS. The map was established by MapInfo Professional 15.0. The live adults were drowned in water for 12-24 hours, and then killed in hot water. Soft bodies were preserved in $95 \%$ ethanol and stored at $-20{ }^{\circ} \mathrm{C}$. Empty shells were cleaned and preserved at room temperature in the Key Laboratory of Molluscan Quarantine and Identification of Fuzhou Customs District, Fujian, China (GACC).

Shells were measured to $0.1 \mathrm{~mm}$ using electronic calipers. Standard shell parameters were taken following Dillon (1984). All adult specimens of each species were measured. Live sexually mature specimens were dissected for the examination of reproductive system under a dissecting microscope (ZEISS Stemi 2000). Terminology for reproductive system follows Gómez (2001). The basal direction starts from the reproductive opening while that of verge starts from the epiphallus following Hwang et al. (2018).

Approximately $30 \mathrm{mg}$ of the foot muscle was used for DNA extraction. The foot muscle was bathed in sterile water for 3-6 hours to remove residual alcohol. Genomic DNA was isolated using Qiagen DNeasy Blood \& Tissue kit (Qiagen, Beijing), examined by agarose gel electrophoresis and ultra-micro spectrophotometer (Implen NP80, Germany), then stored at $-20^{\circ} \mathrm{C}$ for further use. The partial mitochondrial cytochrome c oxidase subunit 1 (COI) was amplified by PCR using apt primer pairs, reaction system, and amplification condition listed in Table 1 . The PCR products were analyzed using $1.2 \%$ agarose gel electrophoresis.

After sequencing, raw sequences were proof-read on chromatograms and aligned into contigs using BioEdit 7.2 (Hall 1999). Sequence alignments were generated using ClustalW implemented in MEGA6 (Tamura et al. 2013). A total of 35 sequences were used in this study, 23 sequences of which were newly generated and deposited in GenBank (Table 2), and the remainder referenced in Wu et al. (2008), Ding et al. (2016), Ai et al. (2016), and Hu et al. (2019). Pairwise $p$-distances between taxa were calculated using MEGA6 (Tamura et al. 2013) and were compared with the currently known intra and inter- specific differentiation values (p-distances) of Camaenidae (Criscione and Köhler 2014; Ai et al. 2016; Ding et al. 2016). Neighbor-Joining and Minimum-Evolution analyses based on COI sequences were performed using MEGA6 (Tamura et al. 2013). Amphidromus atricallosus (Gould, 1843) (Camaenidae) was used 


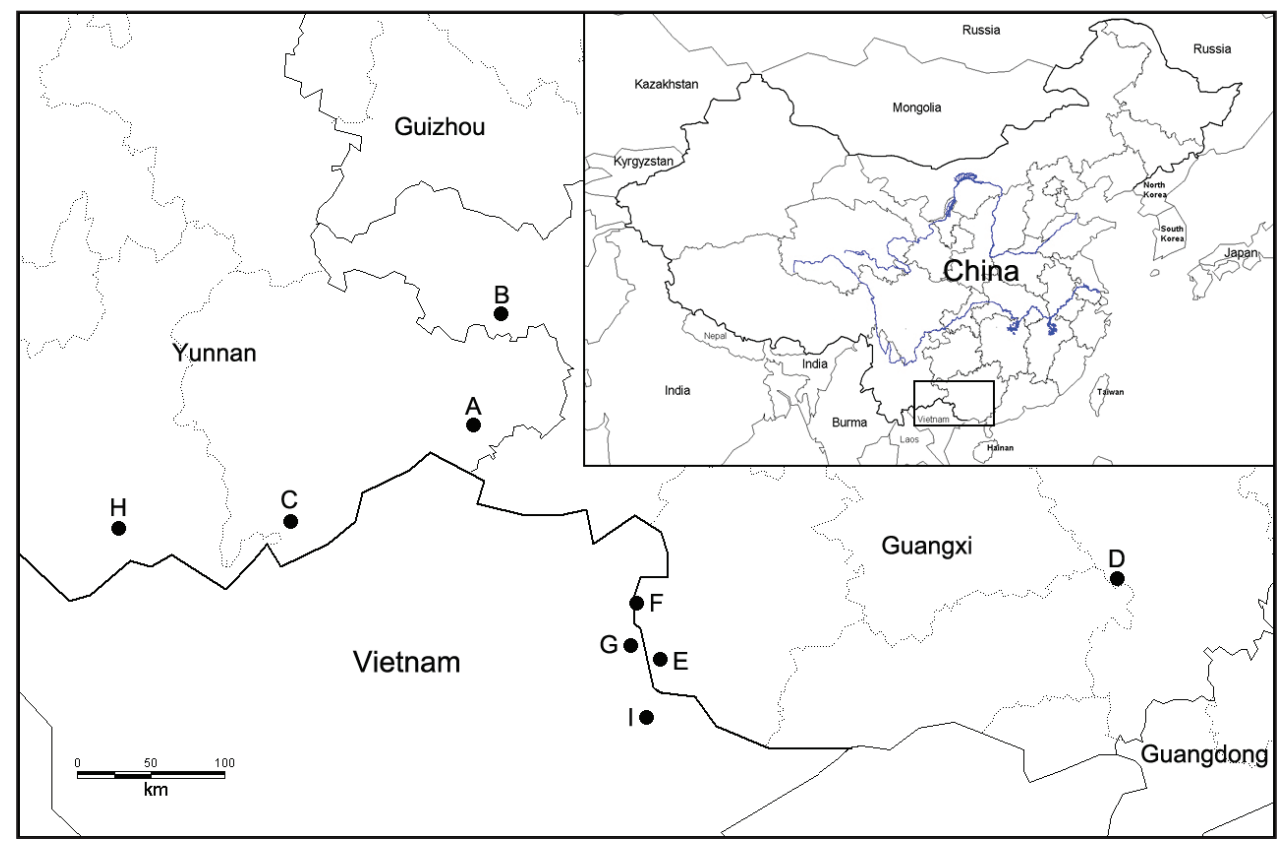

Figure I. Map of locations of Camaena species. C. funingensis sp. nov. A Laolida, Funing, Wenshan, Yunnan, China. C. gaolongensis sp. nov. B Dayao, Gaolong, Tianlin, Guangxi, China. C. maguanensis sp. nov. C Huazhige, Maguan, Wenshan, Yunnan, China. C. yulinensis sp. nov. D Longquan cave, Yulin, Guangxi, China. C. vorvonga E Pingxiang, Guangxi, China F Longzhou, Guangxi, China G That-khe, Vietnam (Type locality). C. jinpingensis $\mathbf{H}$ Jinping, Yunnan, China. C. longsonensis I Lang-Son, Vietnam.

Table I. Primer pairs and PCR conditions used in the analyses of the COI gene of Camaena.

\begin{tabular}{|c|c|}
\hline Gene & COI \\
\hline \multirow[t]{2}{*}{ Primer pairs (5'-3') } & LCO:GGTCAACAAATCATAAAGATATTGG \\
\hline & HCO:TAAACTTCAGGGTGACCAAAAAATCA \\
\hline Reaction systems & $25 \mu \mathrm{l}$ Taq PCR MasterMix $\times 2 ; 1 \mu \mathrm{l}$ each primer; $2 \mu \mathrm{l}$ DNA; $16 \mu \mathrm{lddH_{2 } \mathrm { O }}$ \\
\hline Cycling conditions & $94^{\circ} \mathrm{C}: 30 \mathrm{~s} ; 94^{\circ} \mathrm{C}: 10 \mathrm{~s}, 45^{\circ} \mathrm{C}: 50 \mathrm{~s}, 72^{\circ} \mathrm{C}: 1 \mathrm{~min}, 40$ cycles; $72^{\circ} \mathrm{C}: 10 \mathrm{~min}$. \\
\hline Reference & Folmer et al. 1994 \\
\hline
\end{tabular}

as outgroup. The node support values were assessed by bootstrap resampling using 1000 replicates (Felsenstein 1985).

Abbreviations used in this work:
AG
albumen gland;
F flagellum;
AH aperture height;
FJIQBC Original Fujian Entry-Exit In-
AW aperture width; spection \& Quarantine Bureau,
BC bursa copulatrix; Fuzhou, Fujian, China;
COI cytochrome c oxidase subunit GACC General Administration of 1gene;
E epiphallus;
Customs, People's Republic of China; 


$\begin{array}{llll}\text { HD } & \text { hermaphroditic duct; } & \text { PBC } & \text { pedunculus of bursa copulatrix; } \\ \text { ME } & \text { Minimum-Evolution; } & \text { PR } & \text { penis retractor muscle; } \\ \text { MNHN } & \text { Muséum national d'Histoire } & \text { SH } & \text { shell height; } \\ & \text { naturelle, Paris, France; } & \text { SW } & \text { shell width; } \\ \text { NJ } & \text { Neighbor-Joining; } & \text { V } & \text { verge; } \\ \mathbf{O} & \text { oviduct; } & \text { Va } & \text { vagina; } \\ \mathbf{P} & \text { penis; } & \text { VD } & \text { vas deferens. }\end{array}$

\section{Results}

\section{Molecular analysis}

In this study, a total of 35 sequences of COI from 28 species were used, including eleven sequences from $C$. funingensis sp. nov., C. gaolongensis sp. nov., C. maguanensis sp. nov., and C. yulinensis sp. nov., 8 sequences from sinistral Camaena (C. cicatricosa, C. obtecta, C. inflata, C. connectens, C. hahni, C. detianensis, C. lingyunensis, C. poyuensis), 16 sequences from dextral Camaena and one outgroup (A. atricallosus Family Camaenidae) listed in Table 2.

Table 2. Sampling GenBank accession numbers used in phylogenetic analysis.

\begin{tabular}{|c|c|c|}
\hline Species & COI accession numbers & References \\
\hline Camaena funingensis sp. nov. & MT449465, MT449466, MT449467 & Present study \\
\hline Camaena gaolongensis sp. nov. & MT449468, MT449469, MT449470 & Present study \\
\hline Camaena maguanensis sp. nov. & MT449471, MT449472 & Present study \\
\hline Camaena yulinensis sp. nov. & MT449473, MT449474, MT449475 & Present study \\
\hline Camaena vorvonga & MT984239 & Present study \\
\hline Camaena xanthoderma & MT984235 & Present study \\
\hline Camaena xanthoderma polyzona & MT984236 & Present study \\
\hline Camaena hainanensis & MT984234 & Present study \\
\hline Camaena choboensis & MT984240 & Present study \\
\hline Camaena gabriellae & MT984241 & Present study \\
\hline Camaena gabriellae platytaenia & MT984242 & Present study \\
\hline Camaena longsonensis & EF057379 & Wu et al. 2008 \\
\hline Camaena jinpingensis & KU586503 & Ding et al. 2016 \\
\hline Camaena menglunensis & KU586506 & Ding et al. 2016 \\
\hline Camaena inflata & KU586524 & Ding et al. 2016 \\
\hline Camaena obtecta & KU055610 & Ding et al. 2016 \\
\hline Camaena hahni & KX621263 & Ai et al. 2016 \\
\hline Camaena connectens & KU586518 & Ding et al. 2016 \\
\hline Camaena poyuensis & KU061273 & Ding et al. 2016 \\
\hline Camaena lingyunensis & KX345077 & Ai et al. 2016 \\
\hline Camaena cicatricosa & KU061276 & Ding et al. 2016 \\
\hline Camaena detianensis & KX345074 & Ai et al. 2016 \\
\hline Camaenella platyodon & МH362759 & Hu et al. 2019 \\
\hline Camaena leonhardti & MT984237 & Present study \\
\hline Camaena vulpis & MT984238 & Present study \\
\hline Camaena hemiclista & MT984243 & Present study \\
\hline Camaena haematozona & MT984244 & Present study \\
\hline Amphidromus atricallosus & MT984245 & Present study \\
\hline
\end{tabular}




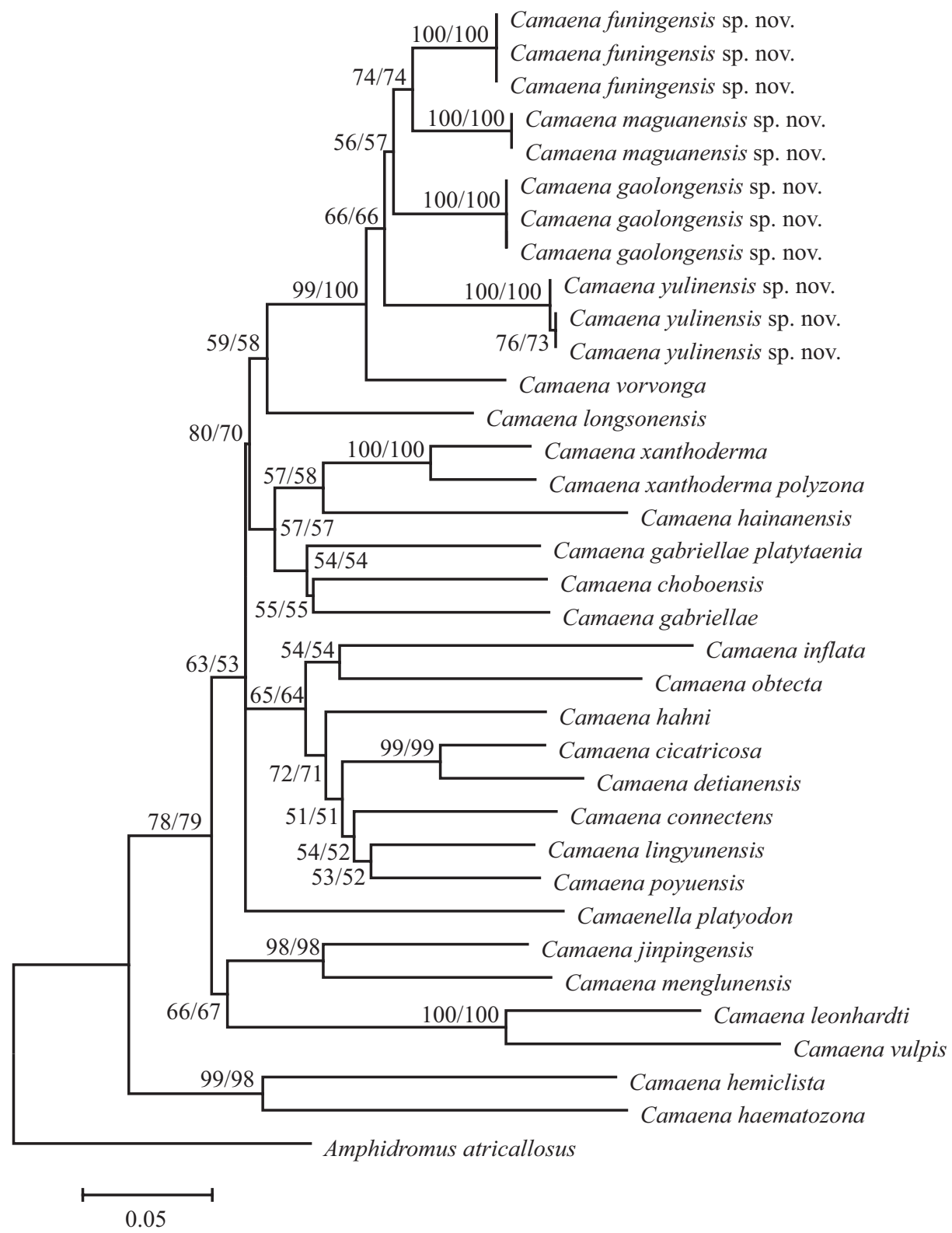

Figure 2. Neighbor-Joining and Minimum-Evolution trees based on analysis of the COI sequences. Numbers beside nodes indicate bootstrapping support (\%) for the main clades, based on 1000 replicates.

Inter and intra-specific $P$-distances from COI gene of seven species were calculated and are listed in Table 3. According to the results of the target gene COI, the $p$-distances between $C$. funingensis sp. nov., C. gaolongensis sp. nov., C. maguanensis sp. nov., and C. yulinensis sp. nov. and other dextral Camaena were 0.068-0.200, 0.075-0.203, $0.068-0.198$ and $0.092-0.202$ respectively. 
Table 3. Inter and intra-specific $P$-distances of the COI sequences on dextral Camaena species.

\begin{tabular}{lcc}
\hline \multicolumn{1}{c}{ Sampling } & \multicolumn{2}{c}{ P-distances } \\
\cline { 2 - 3 } & Within & Between \\
\hline Camaena funingensis sp. nov. & 0.000 & $0.068-0.200$ \\
Camaena gaolongensis sp. nov. & 0.000 & $0.075-0.203$ \\
Camaena maguanensis sp. nov. & 0.000 & $0.068-0.198$ \\
Camaena yulinensis sp. nov. & $0.000-0.002$ & $0.092-0.202$ \\
Camaena vorvonga & $0.000-0.002$ & $0.089-0.209$ \\
Camaena jinpingensis & $0.000-0.002$ & $0.196-0.209$ \\
Camaena longsonensis & 0.000 & $0.153-0.211$ \\
\hline
\end{tabular}

For phylogenetic analysis, results showed that Neighbor-Joining and MinimumEvolution trees had mostly the same topological structure (Fig. 2), and indicated that phylogenic analyses were relatively correct and reliable. The bootstrap support of each species exceeds $50 \%$. The sinistral camaenids were clearly clustered together. The four dextral new species have the closest phylogenetic relationship to each other and are sister species with $C$. vorvonga. From the tree structure, branch length and comparison of the known species, the phylogenetic trees supported $C$. funingensis sp. nov., C. gaolongensis sp. nov., C. maguanensis sp. nov., and C. yulinensis sp. nov. as new species. Moreover, the four new species all had a closer genetic relationship with each other than with any other Camaena species studied here.

\section{Systematics}

\section{Camaenidae Pilsbry, 1895}

\section{Camaena Albers, 1850}

Type species. Helix cicatricosa Müller, 1774, subsequent designation by Martens 1860 .

\section{Camaena funingensis Zhou, Wang \& Lin, sp. nov.}

http://zoobank.org/E94E735E-BAD1-4D8C-AC91-5D50DF90AFE5

Figures 3A, 4, 5A, 6, Tables 3-5

Type material. Holotype. [FJIQBC 19340] Shell height $21.0 \mathrm{~mm}$, shell width $41.0 \mathrm{~mm}$, height of aperture $14.0 \mathrm{~mm}$, width of aperture $18.7 \mathrm{~mm}, 22$ October 2014, collected from the type locality.

Paratype. [FJIQBC 19341-19343] 3 live specimens: 2 adults, 1 juvenile.

Type locality. Laolida, Funing, Wenshan, Yunnan, China (2331'48.88"N, $\left.105^{\circ} 32^{\prime} 59.70 " \mathrm{E}\right)$.

Etymology. The name of the new species refers to the type locality.

Diagnosis. Shell. Shell dextral, large, thin, fragile and lucent, low, and flat conical. 4.5 whorls, the front whorls increasing slowly. Spire relatively low. Body whorl rapidly 

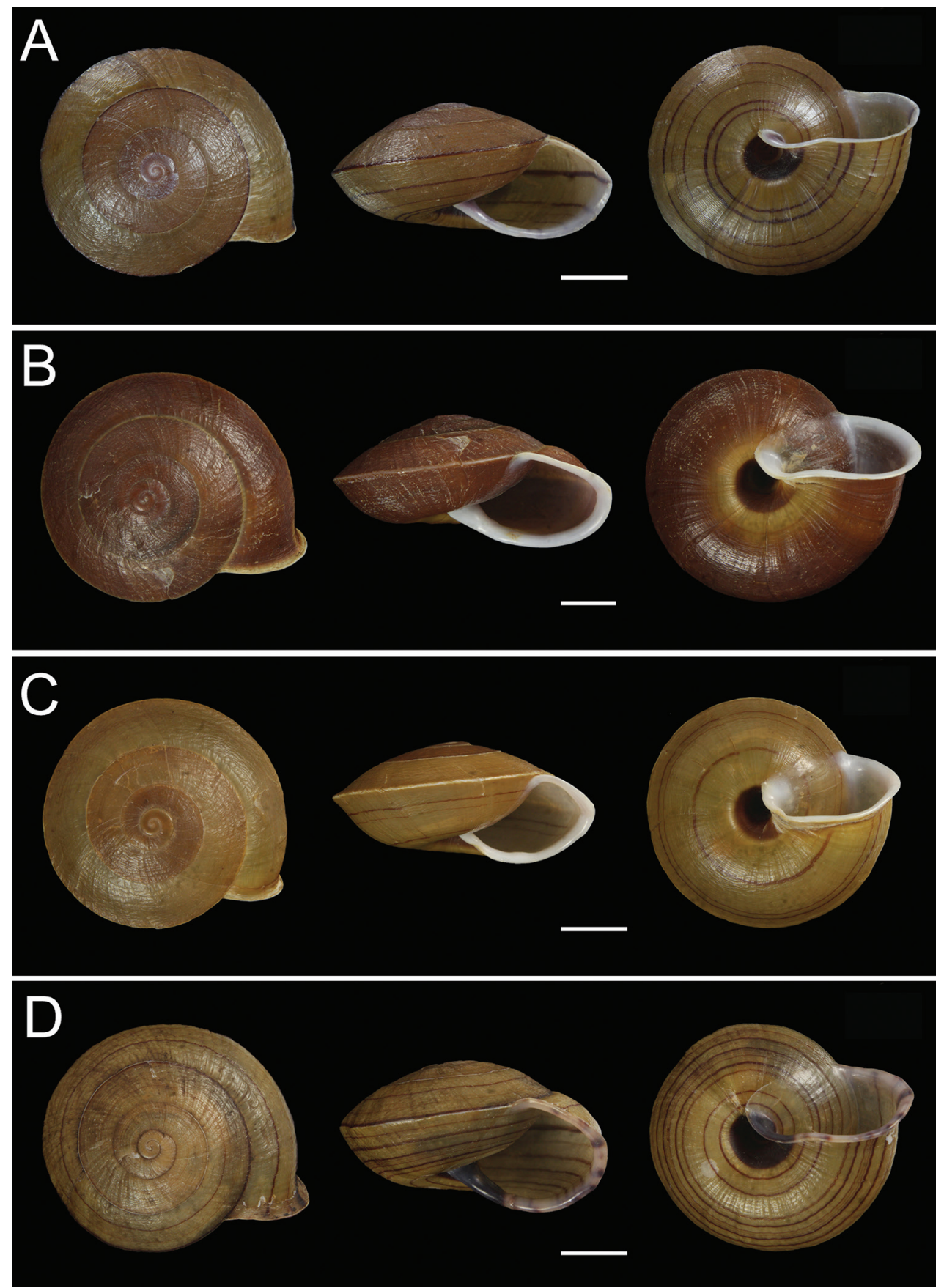

Figure 3. Photographs of the four new species A Camaena funingensis sp. nov. (holotype, FJIQBC 19340, Laolida, Funing, Yunnan, China) B Camaena gaolongensis sp. nov. (holotype, FJIQBC 19353, Dayao, Gaolong, Guangxi, China) C Camaena maguanensis sp. nov. (FJIQBC 19405, Huazhige, Maguan, Yunnan, China) D Camaena yulinensis sp. nov. (FJIQBC 19460, Longquan cave, Yulin, Guangxi, China). Scale bars: $10 \mathrm{~mm}$. 
expanded. Shell light yellowish brown with clear growth lines and spiral bands on the surface. Apex quite blunt. Suture shallow. The protoconch surface smooth, and some short clear growth lines near the inner side of suture under $32 \times$ stereomicroscope. Body whorl with carinate periphery, and a thin reddish brown band on the carina and several sparse bands below the carina. Aperture lunate, slightly descending. Peristome reflected, white, thin, sharp. Columellar lip reflected. Umbilicus reddish brown, large, only $1 / 5$ covered. Inner lip attached to the body whorl, forming translucent callus.

Soft body. Yellowish brown with irregular black lines and spots. Tentacles dark.

Reproductive system. Bursa copulatrix oval and large with long and tapering pedunculus, expanded at the base. Flagellum long, tapering distally. Vas deferens short and thin. Epiphallus long, slightly thick. Penis retractor muscle medium length and slender, becoming wider at the end. Penis swollen and long, with longitudinal, slightly corrugated, strong and widely spaced pilasters internally. Verge ovate, opened terminally, and one clear crack on the verge surface extending from the terminal to the base.

Habitat. The species was found on limestone.

Distribution. Only known from the type locality.

Remarks. Camaena funingensis sp. nov. is characterized by a more oblate shape, lower spire, thin and fragile shell, and yellowish brown coloration, which are clearly different from the other dextral camaenids except $C$. longsonensis (Morlet, 1891), C jinpingensis Chen, Zhang \& Li, 1990, and C. vorvonga (Bavay \& Dautzenberg, 1900) (Chen et al. 1990; Schileyko 2011). The shells of the above four species are distinct from $C$. funingensis in the following ways:

(1) The umbilicus of $C$. funingensis sp. nov. is only $1 / 5$ covered, while that of $C$. longsonensis is almost covered by reflected columellar lip leaving only a narrow slit, and that of $C$. jinpingensis is fully covered.

(2) C. funingensis sp. nov. has several reddish brown bands at the bottom of the body whorl in addition to those on the carina, while only one thin reddish brown band is present on the carinate periphery of $C$. vorvonga.

(3) For C. funingensis sp. nov., the verge is ovate and has one clear crack on the surface extending to the base, which makes it stand out other dextral camaenids.

Camaena gaolongensis sp. nov. is distinguishable from $C$. funingensis sp. nov. in having no spiral band. For $C$. maguanensis sp. nov., there is no band on the carinate periphery of the body whorl except for several below the carina. Moreover, the verge of $C$. maguanensis sp. nov. is small and circular. Camaena yulinensis sp. nov. differs to C. funingensis sp. nov. in having a conical verge and flesh-colored peristome.

$P$-distances of the COI gene between $C$. funingensis sp. nov. and the other camaenids are 0.068-0.200 (Table 3), and those between $C$. funingensis sp. nov. and C. gaolongensis sp. nov., $C$. maguanensis sp. nov. and $C$. yulinensis sp. nov. are 0.075, 0.068 and 0.094 respectively. All of these $P$-distances exceed the maximum intra-specific value 0.059 in the family Camaenidae. On the phylogenetic tree, these four new species are adjacent, hence it is reasonable to designate this as a new species. 


\section{Camaena gaolongensis Zhou, Wang \& Lin, sp. nov.}

http://zoobank.org/1B657A19-59B9-46D2-B874-9DB7120730E9

Figures 3B, 4, 5B, 7, Tables 3-5

Type material. Holotype. [FJIQBC 19353] Shell height $23.8 \mathrm{~mm}$, shell width $49.0 \mathrm{~mm}$, height of aperture $14.0 \mathrm{~mm}$, width of aperture $19.2 \mathrm{~mm}, 11$ April 2015, collected from the type locality.

Paratype. [FJIQBC 19354] 1 live juvenile, 20 October 2014; [FJIQBC 1935519356] 2 live adults, 11 April 2015.

Type locality. Dayao, Gaolong, Tianlin, Guangxi, China $\left(24^{\circ} 11^{\prime} 52.33^{\prime N}\right.$, $\left.105^{\circ} 43^{\prime} 40.56 " \mathrm{E}\right)$.

Etymology. The name of the new species refers to the type locality.

Diagnosis. Shell. Shell dextral, large, thick, strong, low, and flat conical. 4.5 whorls, the front whorls increasing slowly. Spire relatively low. Body whorl rapidly expanded. Shell dark brown with clear and dense growth lines on the surface. Apex quite blunt. Suture shallow. The protoconch surface smooth with scale marks, and some short growth lines clear near the outer side of suture under $32 \times$ stereomicroscope. Body whorl with acute and carinate periphery, but no spiral band. Aperture Ushaped. Peristome reflected, white and thick. Columellar lip reflected. Umbilicus reddish brown, open, large, and only $2 / 5$ covered. Inner lip attached to the body whorl, forming translucent callus.

Soft body. Brown with irregularly black lines and spots. Tentacles dark.

Reproductive system. Bursa copulatrix oval and medium sized with long pedunculus, expanded at the base, becoming thinner at the distal end. Flagellum long and smooth, tapering distally. Vas deferens long and thin. Epiphallus medium length and thick. Penis retractor muscle short, slender basally but wide and flat distally. Penis thick and medium length. Inner penial wall supporting longitudinal, stronger, and more widely spaced pilasters, smooth basally, curved distally. Verge irregularly conical, opened basally, extending from the base to the end, with several slanted wrinkles on the surface.

Habitat. It is common in primary forest and loess areas, but it has not been found on the reclaimed lands outside the primary forest.

Distribution. Only known from the type locality.

Remarks. Camaena gaolongensis sp. nov. is clearly different from other dextral camaenids by its quite thick, low, flat, and dark brown conical shell resembling a flying saucer (Chen et al. 1990, Schileyko 2011). Additionally, the longitudinal pilasters on the inner penial wall are stronger and more widely spaced, as well as smooth at the base but curved at the end, which are also distinct from the other dissected Camaena snails (Ding et al. 2016, Ai et al.2016).

$P$-distances of the COI gene between C. gaolongensis sp. nov. and other dextral Camaena species are 0.075-0.203 (Table 3), and those between C. gaolongensis sp. nov., C. maguanensis sp. nov., and C. yulinensis sp. nov. are 0.085 and 0.104 respectively. Combining the topological structure of the phylogenetic tree, the new species C. gaolongensis sp. nov. is distinct from other dextral Camaena species. 

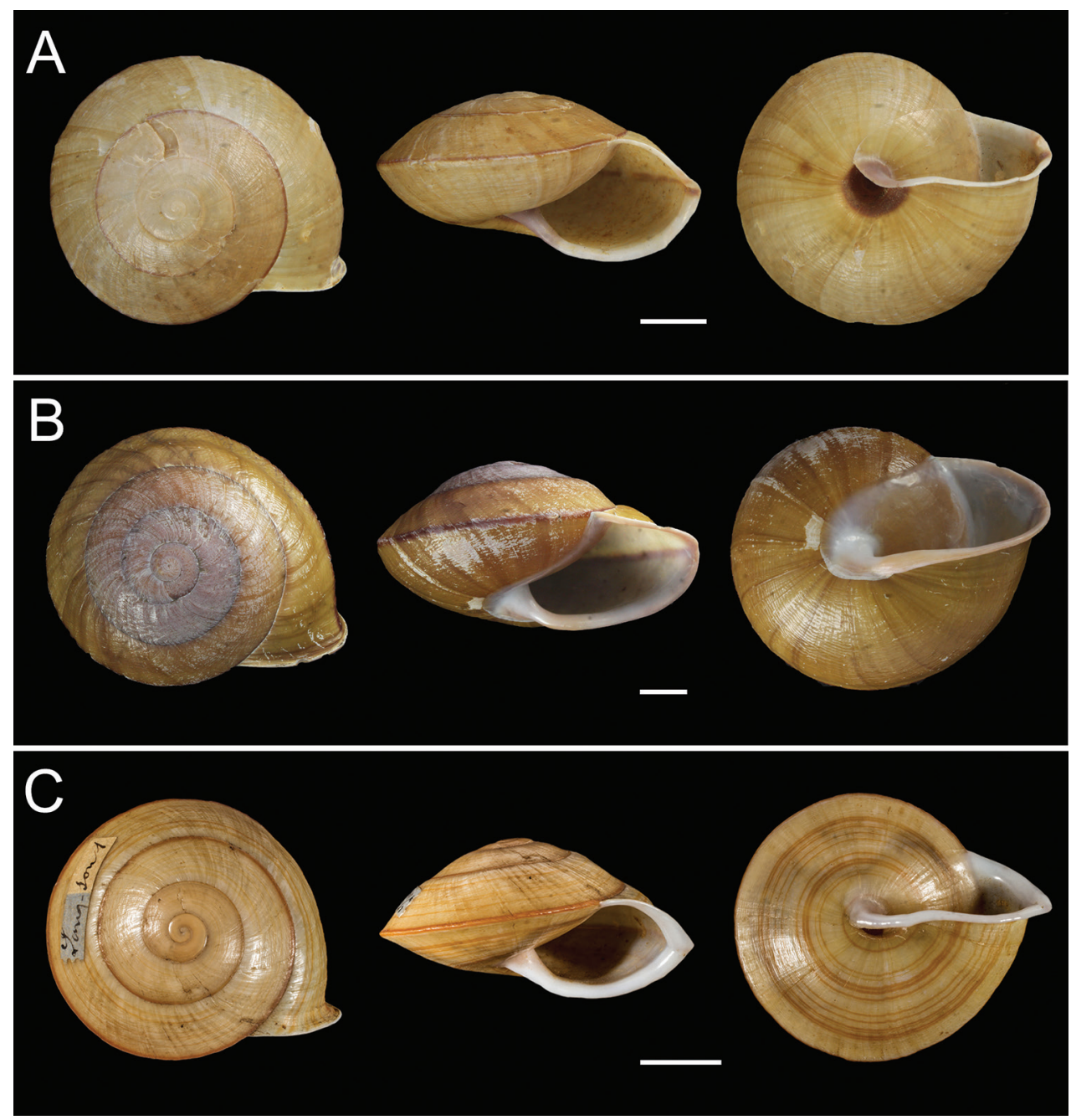

Figure 4. Photographs of three camaenids A Camaena vorvonga (Pingxiang, Guangxi, China) B Camaena jinpingensis (Jinping, Yunnan, China) C Camaena longsonensis (Lang-Son, Vietnam). Scale bars: $10 \mathrm{~mm}$.

Camaena maguanensis Zhou, Wang \& $\mathrm{Hu}$, sp. nov. http://zoobank.org/EC5431C5-CFB6-4309-80C1-0CF8F3C9BE0E Figures 3C, 4, 5C, 8, Tables 3-5

Type material. Holotype. [FJIQBC 19405] Shell height $19.2 \mathrm{~mm}$, shell width $39.0 \mathrm{~mm}$, height of aperture $12.0 \mathrm{~mm}$, width of aperture $16.5 \mathrm{~mm}, 16$ April 2015, collected from the type locality.

Paratype. [FJIQBC 19406] 1 live adult; [FJIQBC 19407-19413] 7 empty shells: 5 adults, 2 juveniles.

Type locality. Huazhige, Maguan, Wenshan, Yunnan, China (2257'24.48"N, $104^{\circ} 21^{\prime} 12.96^{\prime \prime E) . ~}$ 

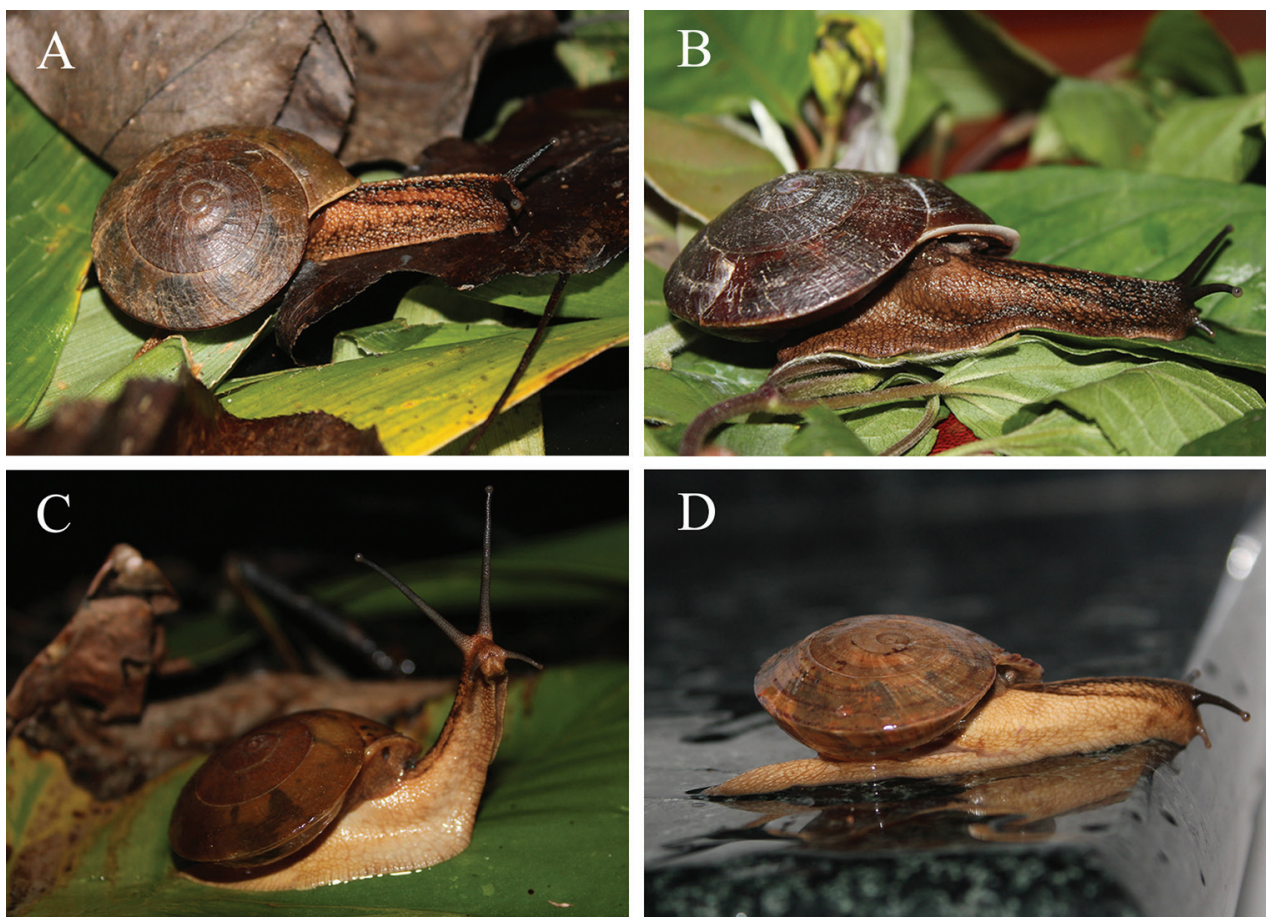

Figure 5. Ecological photographs of snails A Camaena funingensis sp. nov. (Laolida, Funing, Yunnan, China) B Camaena gaolongensis sp. nov. (Dayao, Gaolong, Guangxi, China) C Camaena maguanensis sp. nov. (Huazhige, Maguan, Yunnan, China) D Camaena yulinensis sp. nov. (Longquan cave, Yulin, Guangxi, China).

Table 4. Adult shell dimensions (mm).

\begin{tabular}{lcccc}
\hline \multicolumn{1}{c}{ Species } & C. funingensis sp. nov. & C. gaolongensis sp. nov. & C. maguanensis sp. nov. & C. yulinensis sp. nov. \\
\hline Voucher & FJIQBC19340-19342 & FJIQBC19353 & FJIQBC19405-19411 & FJIQBC19460-19466 \\
& & FJIQBC19355-19356 & & FJIQBC19468-19470 \\
Sample size & 3 & 3 & 7 & 10 \\
SH & $19.5-21.0$ & $23.5-24.5$ & $19.2-22.0$ & $19.8-23.0$ \\
& $(20.17 \pm 0.62)$ & $(23.93 \pm 0.42)$ & $(20.36 \pm 0.90)$ & $(21.35 \pm 1.05)$ \\
SW & $39.2-41.0$ & $47.0-50.0$ & $38.0-40.5$ & $37.0-42.6$ \\
& $(40.23 \pm 0.76)$ & $(48.67 \pm 1.25)$ & $(39.24 \pm 0.74)$ & $(40.54 \pm 1.58)$ \\
SW/SH & $1.95-2.03$ & $2.00-2.06$ & $1.84-2.03$ & $1.84-1.96$ \\
& $(2.00 \pm 0.03)$ & $(2.03 \pm 0.02)$ & $(1.93 \pm 0.06)$ & $(1.90 \pm 0.03)$ \\
AH & $13.4-14.0$ & $13.8-14.2$ & $12.0-13.1$ & $13.0-14.6$ \\
& $(13.63 \pm 0.26)$ & $(14.00 \pm 0.16)$ & $(12.64 \pm 0.34)$ & $(13.76 \pm 0.48)$ \\
AW & $18.0-18.7$ & $19.0-19.4$ & $16.5-18.1$ & $17.5-21.6$ \\
& $(18.30 \pm 0.29)$ & $(19.20 \pm 0.16)$ & $(17.22 \pm 0.56)$ & $(19.11 \pm 1.46)$ \\
AW/AH & $1.34-1.35$ & $1.37-1.38$ & $1.33-1.39$ & $1.33-1.48$ \\
& $(1.34 \pm 0.01)$ & $(1.37 \pm 0.00)$ & $(1.36 \pm 0.02)$ & $(1.39 \pm 0.06)$ \\
\hline
\end{tabular}

Etymology. The name of the new species refers to the type locality.

Diagnosis. Shell. Shell dextral, large, thin, fragile, and glossy, low and flat conical. 4.5 whorls, the front whorls increasing slowly. Spire relatively low. Body whorl rapidly expanded. Shell yellowish with unclear growth lines and spiral bands on the surface. 
Apex quite blunt. Suture shallow. The protoconch surface smooth, some short growth lines visible near the two sides of suture under $32 \times$ stereomicroscope. Last whorl with quite acute carina at periphery and a shallow groove-like depression above and below the carina. No band on the carina, but several reddish brown and sparse spire bands below the carina. Aperture crescent-shaped. Peristome reflected, white and thick. Columellar lip reflected. Umbilicus reddish brown, open, large and only 2/5 covered. Inner lip attached to the body whorl, forming translucent callus.

Soft body. Light yellowish brown with black lines. Tentacles dark.

Reproductive system. Bursa copulatrix oval, small, with quite long and tapering pedunculus. Flagellum long, tapering distally. Vas deferens long and thin. Epiphallus medium thickness and length. Penis retractor muscle very short and slender. Penis long with a short protrusion at the middle. Inner penial wall with longitudinal, slightly straight and smooth pilasters. Verge circular, somewhat small, opened basally, extending from the base to the end.

Habitat. The species was found on limestone in Maguan county of Yunnan province, China.

Distribution. Only known from the type locality.

Remarks. Camaena maguanensis sp. nov. is clearly different from other dextral camaenids with a lower conical shell. In particular, C. maguanensis sp. nov. has a large and open umbilicus, which distinguishes it from $C$. longsonensis and $C$. jinpingensis. Although the umbilicus of $C$. maguanensis sp. nov. is similar to that of $C$. vorvonga, some differences are obvious. For example, $C$. maguanensis sp. nov. has no spiral band on the carinate periphery of the body whorl but some spaced bands at the base. The shell of C. maguanensis sp. nov. is yellowish, but that of $C$. gaolongensis sp. nov. is dark brown. On the other hand, $C$. maguanensis sp. nov. has a circular and slightly smaller verge.

$P$-distances of the COI gene between this new species and the other dextral species are 0.068-0.198 (Table 3), and that between C. maguanensis sp. nov. and C. yulinensis sp. nov. is 0.108 , also exceeding 0.059 (currently the maximum differentiation value (p-distance) of Camaenidae) (Criscione and Köhler 2014), and the topology of the phylogenetic tree also supports the new species.

\section{Camaena yulinensis Zhou, Wang \& $\mathrm{Hu}$, sp. nov.}

http://zoobank.org/3038DBDB-A3B2-4364-B2D3-CB7E694EA8ED

Figures 3D, 4, 5D, 9, Tables 3-5

Type material. Holotype. [FJIQBC 19460] Shell height $21.0 \mathrm{~mm}$, shell width $40.5 \mathrm{~mm}$, height of aperture $13.5 \mathrm{~mm}$, width of aperture $18.2 \mathrm{~mm}, 21$ September 2014, collected from the type locality.

Paratype. [FJIQBC 19461-19466] 6 specimens: 3 live adults, 3 empty adult shells, 4 November 2013; [FJIQBC 19468-19472] 5 specimens: 3 live adults, 2 empty juvenile shells, 21 September 2014.

Type locality. Longquan cave, Yulin, Guangxi, China (22³6'41.24"N, $\left.109^{\circ} 45^{\prime} 21.36 " \mathrm{E}\right)$. 


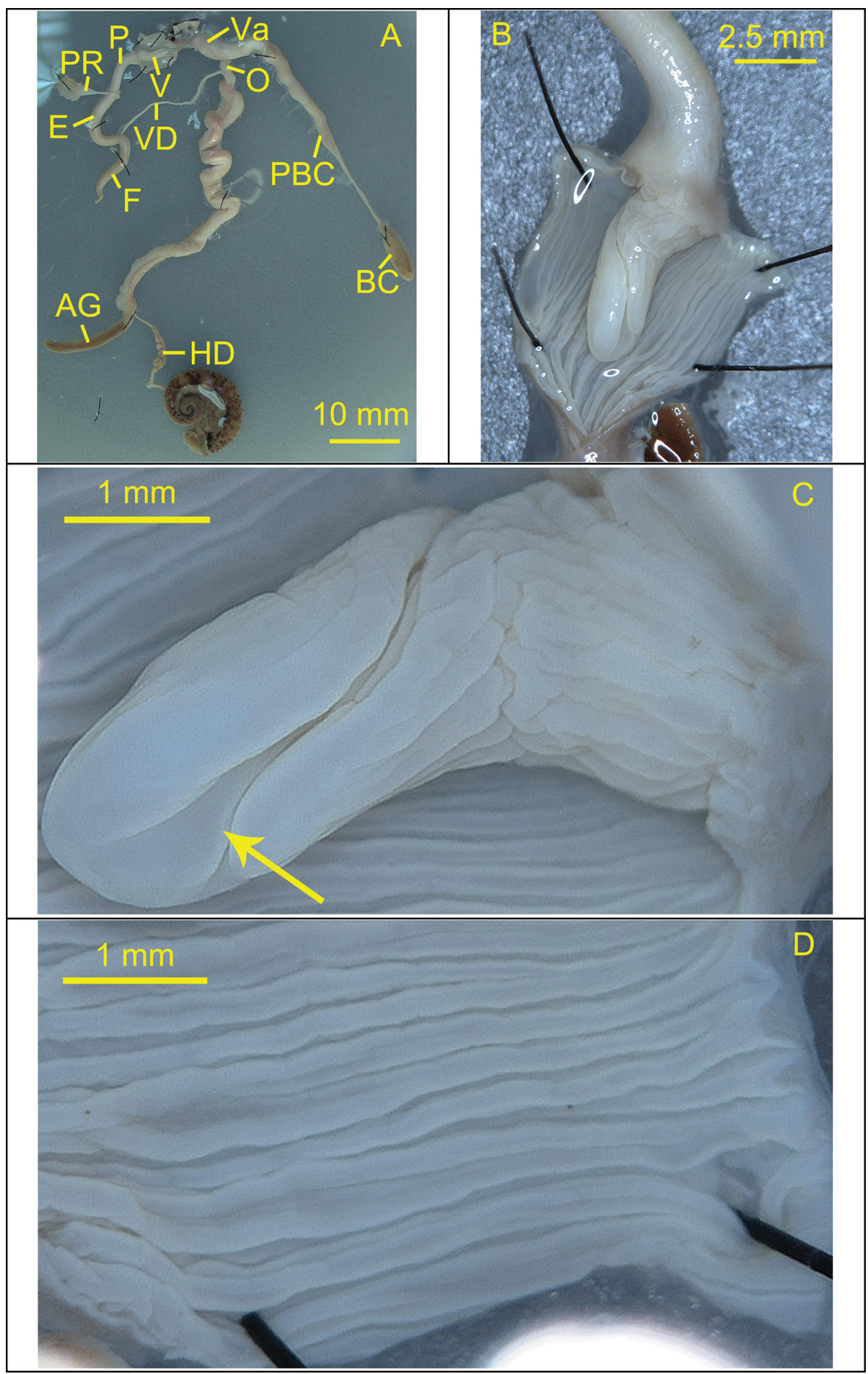

Figure 6. Reproductive system of the snail Camaena funingensis sp. nov. (holotype, FJIQBC 19340, Laolida, Funing, Yunnan, China) A reproductive organ B penis $\mathbf{C}$ verge $\mathbf{D}$ inner penial wall. The arrow indicates opening position of the verge. 


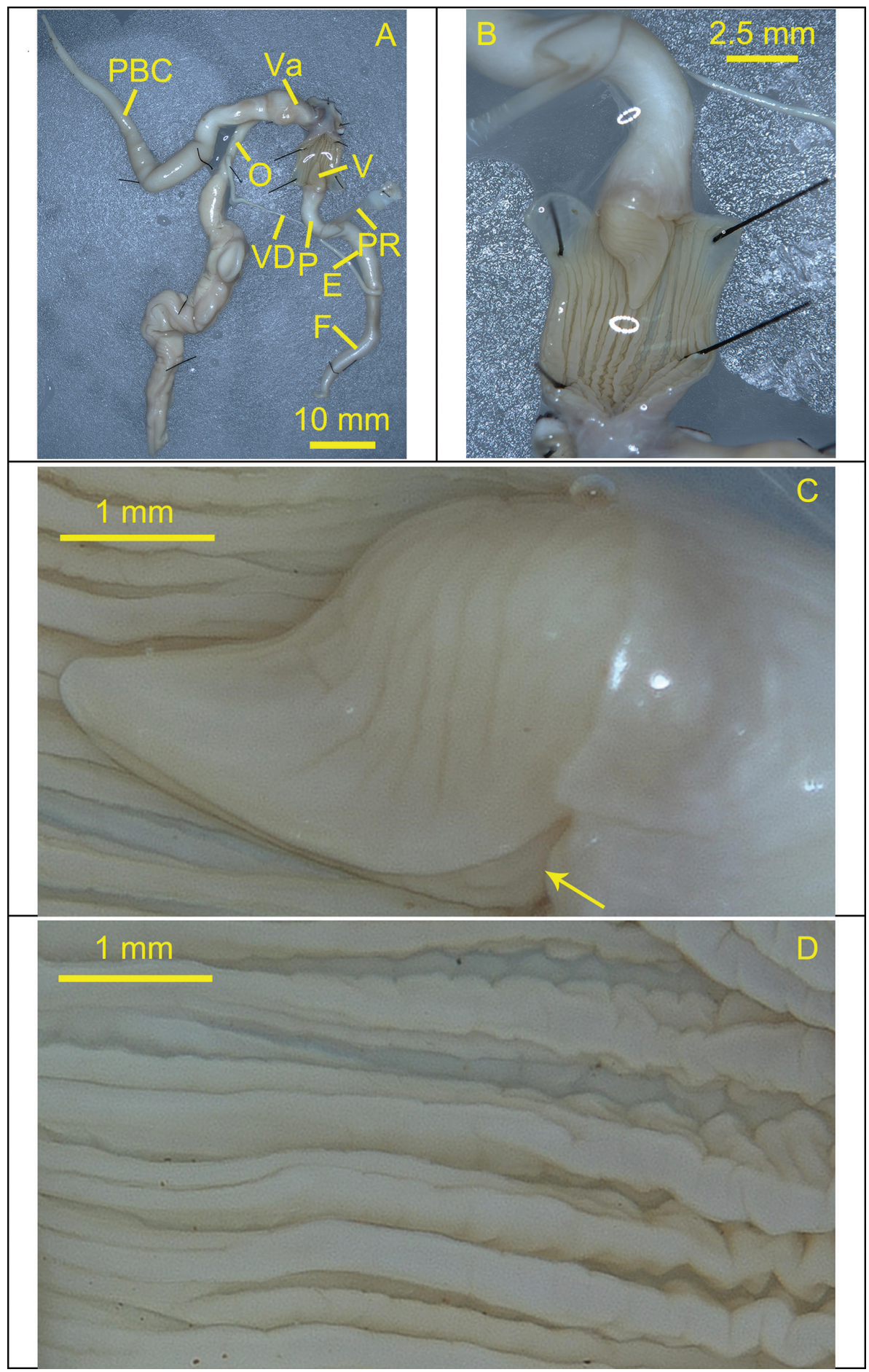

Figure 7. Reproductive system of the snail Camaena gaolongensis sp. nov. (holotype, FJIQBC 19353, Dayao, Gaolong, Guangxi, China) A reproductive organ B penis $\mathbf{C}$ verge $\mathbf{D}$ inner penial wall. The arrow indicates opening position of the verge. 


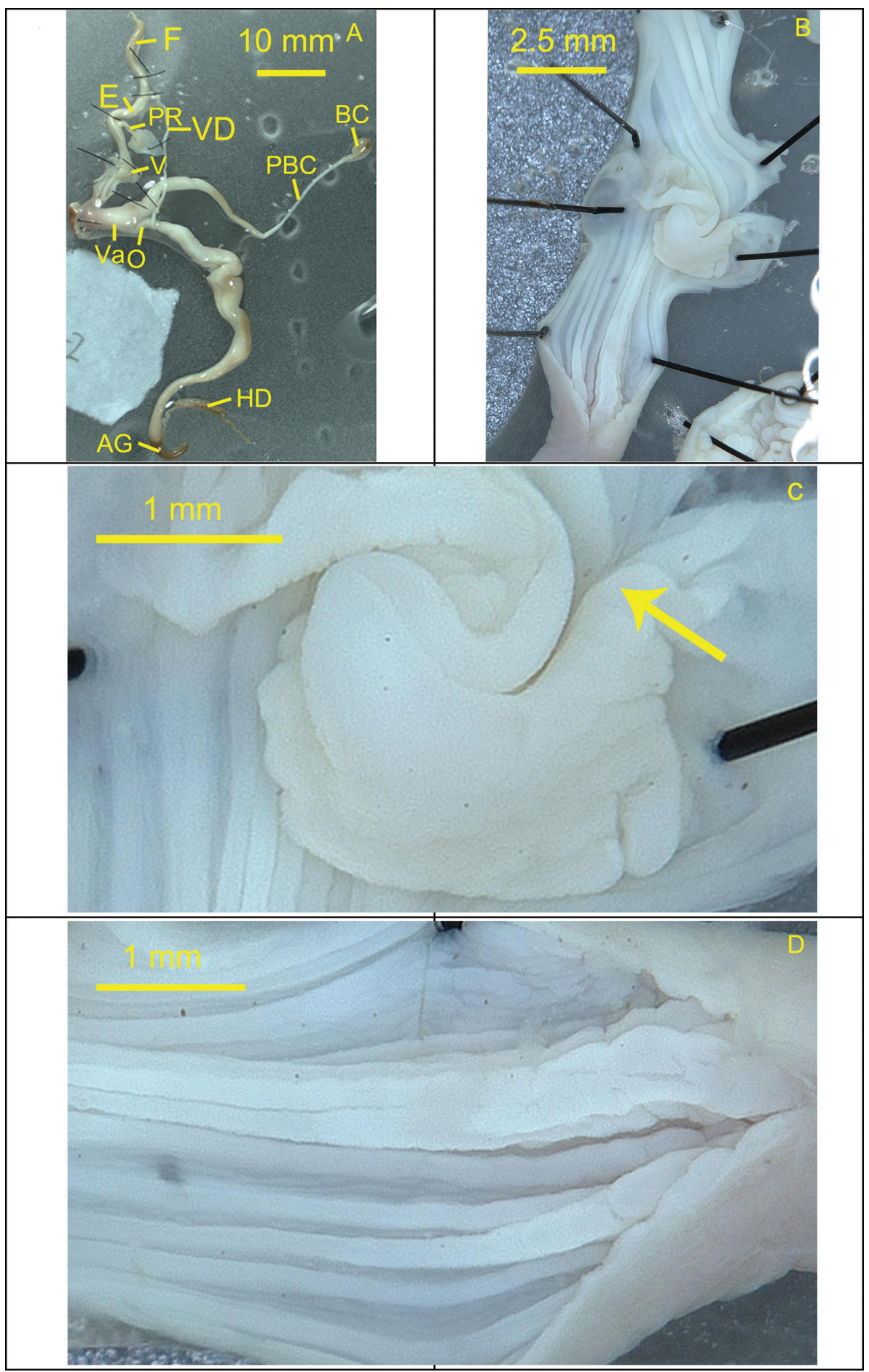

Figure 8. Reproductive system of the snail Camaena maguanensis sp. nov. (FJIQBC 19405, Huazhige, Maguan, Yunnan, China) A reproductive organ $\mathbf{B}$ penis $\mathbf{C}$ verge $\mathbf{D}$ inner penial wall. The arrow indicates opening position of the verge. 


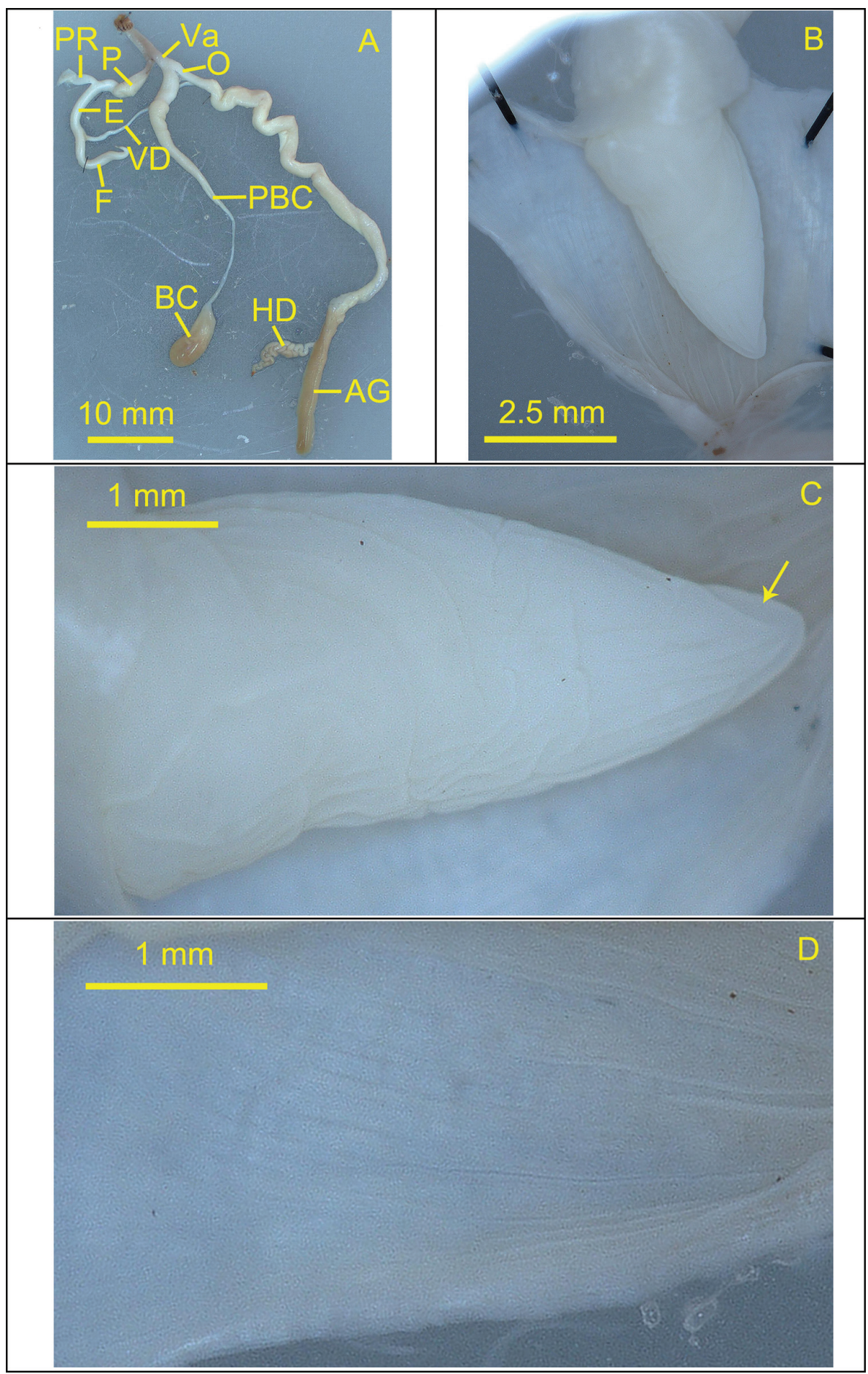

Figure 9. Reproductive system of the snail Camaena yulinensis sp. nov. (FJIQBC 19460, Longquan cave, Yulin, Guangxi, China) A reproductive organ B penis $\mathbf{C}$ verge $\mathbf{D}$ inner penial wall. The arrow indicates opening position of the verge. 
Table 5. Diagnostic comparisons of morphological characters of the four new species.

\begin{tabular}{|c|c|c|c|c|}
\hline Character & C. funingensis sp. nov. & C. gaolongensis sp. nov. & C. maguanensis sp. nov. & C. yulinensis sp.nov. \\
\hline Shell thickness & thin & quite thick & thin & thin \\
\hline Shell color & light yellowish brown & dark brown & yellowish & light yellowish \\
\hline Periphery & carinate & acute and carinate & acute and carinate & carinate \\
\hline Growth lines & clear & clear and dense & unclear & clear and dense \\
\hline Umbilicus & only $1 / 5$ covered & only $2 / 5$ covered & only $2 / 5$ covered & $1 / 3$ covered \\
\hline Verge & ovate & short conic & circular and small & long conic \\
\hline Verge opening & $\begin{array}{l}\text { terminally, one clear crack on } \\
\text { the surface extending from } \\
\text { the end to the base }\end{array}$ & $\begin{array}{l}\text { basally, one crack on the } \\
\text { side surface extending from } \\
\text { the base to the end }\end{array}$ & $\begin{array}{l}\text { basally, one crack on the } \\
\text { surface extending from the } \\
\text { base to the end }\end{array}$ & terminally \\
\hline
\end{tabular}

Etymology. The name of the new species refers to the type locality.

Diagnosis. Shell. Shell dextral, large, thin, fragile, and slightly lucent, low and flat conical. 4.5 whorls, the front whorls increasing slowly. Spire relatively low. Body whorl rapidly expanded. Shell light yellowish with clear and dense growth lines and spiral bands on the surface. Apex quite blunt. Suture shallow. The protoconch surface smooth for most individuals, but a few are rough. Growth lines clear near the outer side of suture under $32 \times$ stereomicroscope. Last whorl with carinate periphery, a thin reddish brown spiral band on the carina, and many reddish brown spiral bands of different thickness on the upper and lower parts. Aperture lunate. Peristome reflected, fleshcolored, thin, sharp. Columellar lip reflected. Umbilicus reddish brown, open, large, and only $1 / 3$ covered. Inner lip attached to body whorl, forming translucent callus.

Soft body. Pale yellow with irregular black lines. Tentacles dark brown.

Reproductive system. Bursa copulatrix oval, large, with long and tapering pedunculus. Flagellum long and slightly thick, tapering distally. Vas deferens short and thin. Epiphallus medium length and slightly thick. Penis retractor muscle short and wide. Penis short and swollen at distal $1 / 3$, with longitudinal, thin, smooth pilasters internally. Verge conical, large, opened terminally, with some irregular wrinkles on the surface.

Habitat. The species was found on limestone in Yulin city, Guangxi province.

Distribution. Only known from the type locality.

Remarks. Camaena yulinensis sp. nov. differs from C. longsonensis and C. jinpingensis in the key characteristic of large open umbilicus. This new species not only has spiral bands with different thickness on the body whorl but also has a flesh-colored peristome compared to $C$. vorvonga. The differences between this species and the other three new Camaena species herein have already been described above.

$P$-distances of the COI gene between $C$. yulinensis sp. nov. and the other dextral congeners ranges from 0.092 to 0.202 (Table 3) and the phylogenetic topology tree supports the establishment of this new species.

\section{Discussion}

We describe four new species of dextral Camaena snails, namely $C$. funingensis sp. nov., C. gaolongensis sp. nov., C. maguanensis sp. nov. and C. yulinensis sp. nov., which 
are distinguished from their congeners by their shell morphologies, especially the low and flat shell shape, the large open umbilicus, the acute and carinate periphery of the body whorl, as well as features in their reproductive systems and molecular characteristics. Among the first three new species, the differences of shells and genitals are obvious. Although $C$. funingensis sp. nov. and C. yulinensis sp. nov. are similar in shell morphology except size, color and umbilicus, the former has an ovate and terminally opened verge and one clear crack on the surface extending from the end to the base, as well as strong and widely spaced penis pilasters, that distinguish it from C. yulinensis sp. nov. with a conical verge, thin penial inner pilasters and without crack on the surface (Figs 3, 5-9). Nonetheless, the two similar-shaped species are relatively distantly related genetically (Fig. 2).

Some scholars have considered genetic distance as one of the more important pieces of evidence used for identifying new species and revising species; for example, in the Asian camaenids Luchuhadra (Kameda et al. 2007) and Satsuma (Wu et al. 2008), the Australian camaenid Kimberleytrachia (Criscione and Köhler 2014), and Camaena (Ai et al. 2016; Ding et al. 2016). In the present study, the $p$-distances between $C$. funingensis sp. nov., C. gaolongensis sp. nov., C. maguanensis sp. nov., C. yulinensis sp. nov., and the other dextral Camaena was substantial: 0.068-0.200, $0.075-0.203,0.068-0.198$, and $0.092-0.202$ respectively for the mitochondrial COI barcoding region (Table 3 ). These numbers exceed the intra-specific differentiation values ( $p$-distances) of Camaenidae (for Camaena, minimum 0.00, maximum 0.018 in Ding et al. (2016), minimum 0.00, maximum 0.019 in Ai et al. (2016), for Kimberleytrachia, minimum 0.00, maximum 0.059, mean 0.026 in Criscione and Köhler (2014). Based on these considerations, inter-specific differentiation supports the recognition of the four new species.

In the phylogenetic analyses, $C$. vorvonga and $C$. longsonensis, which were placed in informal subgeneric group I, have a close relationship, while they are distant from C. jinpingensis that originally also belonged to group I. In the future, more species and sequences will be needed for a more robust analysis of camaenid phylogeny.

During our long-term field investigations, we observed that most Camaena species have a narrow distribution and a low population density, and only inhabit primary forests. An exception to this is C. cicatricosa, which is widespread and has high population densities (Ai et al. 2016; Ding et al. 2016). In recent years, with the development of the Chinese economy, areas of primary forest have been decreasing and the habitats of Camaena species are becoming increasingly restricted and threatened. Therefore, it is necessary to maximize forest protection, prevent deforestation, and prevent excessive tourist development to preserve the biodiversity of these terrestrial mollusks and other animals and plants.

\section{Acknowledgements}

We gratefully acknowledge the assistance of Chung-Chi Hwang (National University of Kaohsiung) in the field work, and the Muséum national d'Histoire naturelle, Paris, 
France, for open access to the digitized photograph of type specimens. This research is supported by National Natural Science Foundation of China (31801960, 31372162), Agricultural Science and Technology Major Project Funds of Fujian (2017NZ0003-1) and National Key Research and Development Program of China (2017YFF0210304).

\section{References}

Adams H (1870) Descriptions of a new genus, and of eighteen new species of Molluscs. Proceedings of the Zoological Society of London 1870: 5-9.

Albers JC (1850) Die Heliceen, Nach Natürlicher Verwandtschaft Systematisch Geordnet. T. C. F. Enslin, Berlin, 262 pp. https://www.biodiversitylibrary.org/page/11965983

Ai HM, Lin JH, Wang P, Zhou WC, Hwang CC (2016) Descriptions of two new species of the genus Camaena from Guangxi, China (Gastropoda, Stylommatophora, Camaenidae). ZooKeys 634: 29-45. https://doi.org/10.3897/zookeys.634.10236

Bavay A, Dautzenberg P (1900) Diagnose de nouveaux mollusques d'Indo-Chine. Journal de Conchyliologie 48: 108-122.

Bavay A, Dautzenberg P (1909) Description de coquilles nouvelles de l'Indo-Chine. Journal de Conchyliologie 57: 163-206.

Chen DN, Gao JX (1987) Economic Fauna Sinica of China (Terrestrial Mollusca). Science Press, Beijing, 186 pp.

Chen DN, Zhang GQ (1999) Studies on the genus Camaena from China, with description of two new species (Gastropoda: Camaenidae). Transactions of the Chinese Society of Malacology 8: 28-43.

Chen DN, Zhang QG, Li BH (1990) A new terrestrial snail species from China. Zoological Systematics 2(6): 33-35.

Criscione F, Köhler F (2014) Molecular phylogenetics and comparative anatomy of Kimberleytrachia Köhler, 2011 - a genus of land snail endemic to the coastal Kimberley, Western Australia with description of new taxa (Gastropoda, Camaenidae). Contributions to Zoology 83(4): 245-267. https://doi.org/10.1163/18759866-08304003

Dautzenberg PH, Fischer H (1906) Liste de mollusques récoltés par M. H. Mansuy en Indochine et au Yunnan, et description d'espèces nouvelles. Journal de Conchyliologie, Paris 53: 343-471.

Dillon RT (1984) What shall I measure on my snails? Allozyme data and multivariate analysis used to reduce the nongenetic component of morphological variance in Goniobasis proxima. Malacologia 25: 503-511.

Ding HL, Wang P, Qian ZX, Lin JH, Zhou WC, Hwang CC, Ai HM (2016) Revision of sinistral land snails of the genus Camaena (Stylommatophora, Camaenidae) from China based on morphological and molecular data, with description of a new species from Guangxi, China. ZooKeys 584: 25-48. https://doi.org/10.3897/zookeys.584.7173

Eddy S (1998) Profile hidden Markov models. Bioinformatics 14: 755-763. https://doi. org/10.1093/bioinformatics/14.9.755

Felsenstein J (1985) Confidence limits on phylogenies: an approach using the bootstrap. Evolution 39: 783-791. https://doi.org/10.2307/2408678 
Fischer H (1898) Notes sur la faune du Haut Tonkin. III. Liste des mollusques recueillis par le Dr A. Billet. Bulletin scientifique de la France et de la Belgique 28: 310-338.

Folmer O, Black M, Hoeh W, Lutz R, Vrijenhoek R (1994) DNA primers for amplification of mitochondrial cytochrome c oxidase subunit I from diverse metazoan invertebrates. Molecular Marine Biology and Biotechnology 3: 294-299.

Gómez BJ (2001) Structure and functioning of the reproductive system. In: Baker GM (Ed.) The Biology of Terrestrial Molluscs. CABI Publishing, Oxon, 307-330. https://doi. org/10.1079/9780851993188.0307

Gould AA (1843) Description of land mollusks from the province of Tavoy, in British Burmah. Proceedings of the Boston Society of Natural History 1843: 137-141.

Gredler V (1888) Zur Conchylien-Fauna von China. XIII. Stück. Jahrbücher der deutschen malakozoologischen Gesellschaft, 14: 343-373.

Hall TA (1999) BioEdit: a user-friendly biological sequence alignment editor and analysis program for Windows 95/98/NT. Nucleic Acids Symposium Series 41: 95-98. https://doi. org/10.1021/bk-1999-0734.ch008

Hoso M, Kameda Y, Wu SP, Asami T, Kato M, Hori M (2010) A speciation gene for left-right reversal in snails results in anti-predator adaptation. Nature Communications 1: e133. https://doi.org/10.1038/ncomms1133

Hu ML, Wang P, Chen Y, Zhang MZ, Yang SP, Lin JH, Zhou WC (2019) The mitochondrial genome of the land snail Camaenella platyodon (Pfeiffer, 1846) (Stylommatophora, Camaenidae), Mitochondrial DNA Part B 4: 2753-2754. https://doi.org/10.1080/23802359.2019.1644559

Hwang CC (2012) Anatomy and taxonomy of Satsuma succincta (Adams, 1866) and Satsuma batanica pancala (Schmacker \& Boettger, 1891) (Gastropoda: Camaenidae) from southern Taiwan. Bulletin of Malacology 35: 1-11.

Hwang CC, Okuba K, Tada A (2018) Satsuma jinlunensis-a new species from Taiwan (Stylommatophora: Camaenidae). Molluscan Research 38: 1-6. https://doi.org/10.1080/132358 18.2017.1358340

Inkhavilay K, Sutcharit C, Bantaowong U, Chanabun R, Siriwut W, Srisonchai R, Polyotha A, Jirapatrasilp P, Panha S (2019) Annotated Checklist of the Terrestrial Molluscs from Laos (Gastropoda: Neritimorpha, Caenogastropoda and Heterobranchia). ZooKeys 834: 1-166. https://doi.org/10.3897/zookeys.834.28800

Kameda Y, Kawakita A, Kato M (2007) Cryptic genetic divergence and associated morphological differentiation in the arboreal land snail Satsuma (Luchuhadra) largillierti (Camaenidae) endemic to the Ryukyu Archipelago, Japan. Molecular Phylogenetics and Evolution 45: 519-533. https://doi.org/10.1016/j.ympev.2007.03.021

Koetschan C, Förster F, Keller A, Schleicher T, Ruderisch B, Schwarz R, Müller T, Wolf M, Schultz J (2010) The ITS2 Database III - sequences and structures for phylogeny. Nucleic Acids Research 38: 275-279. https://doi.org/10.1093/nar/gkp966

Kuroda L, Habe T (1949) Helicacea. Osaka, 129 pp. [1 pl.]

Mabille J (1889) Contribution à la faune malacologique du Tonkin. A. Masson, Meulan, 20 pp.

Minato H (1980) Land snail fauna of Miyako Islands, the southern Ryukyu, Japan. Venus 39: 83-99.

Möllendorff OF von (1882) Diagnoses specierum novarum Chinae meridionalis. Jahrbücher der Deutschen Malakozoologischen Gesellschaft 9: 179-188. 
Möllendorff OF von (1885) Materialien zur Fauna von China. Die Auriculaceen. Jahrbücher der Deutschen Malakozoologischen Gesellschaft 12: 349-398.

Möllendorff OF von (1888) Mittheilungen aus dem Gebiete der Malakozoologie. Diagnoses specierum novarum sinensium. Nachrichtsblatt der deutschen malakozoologischen Gesellschaft 20(2/3): 38-44.

Morlet L (1891) Diagnoses Molluscorum novorum, in Indo-Chinâ collectorum. Journal de Conchyliologie, Paris 39: 25-28.

Müller OF (1774) Vermivm terrestrium et fluviatilium, v.2. Havni.apud Heineck et Faber, 214 pp.

Páll-Gergely B, Hunyadi A, Otani JU, Asami T (2016) An impressive new camaenid, Entadella entadiformis gen. \& sp. n. from Guangxi, China (Gastropoda: Pulmonata). Journal of Conchology 42(4): 167-179.

Palumbi S, Martin A, Romano S, Mcmillan WO, Stice L, Grabowwski G (1991) The Simple Fool's Guide to PCR. Department of Zoology, University of Hawaii, Honolulu, 46 pp.

Pilsbry HA (1893) Preliminary outline of a new classification of the helices. Academy of Natural Sciences of Philadelphia, Philadelphia, 44: 387-404.

Pilsbry HA (1894) Manual of Conchology. Series 2, vol.9. Academy of Natural Sciences, Philadelphia, $302 \mathrm{pp}$.

Richardson L (1985) Camaenidae: catalog of species. Tryonia 12: 1-479.

Schileyko AA (2003) Treatise on recent terrestrial pulmonate molluscs. Part 11. Trigonochlamydidae, Papillodermidae, Vitrinidae, Limacidae, Bielziidae, Agriolimacidae, Boettgerillidae, Camaenidae. Ruthenica Suppl 2: 1510-1621.

Schileyko AA (2011) Check-list of land pulmonate molluscs of Vietnam (Gastropoda: Stylommatophora). Ruthenica 21(1): 1-68.

Tamura K, Stecher G, Peterson D, Filipski A, Kumar S (2013) MEGA6: Molecular Evolutionary Genetics Analysis version 6.0. Molecular Biology and Evolution 30(12): 2725-2729. https://doi.org/10.1093/molbev/mst197

Vaught KC (1989) A Classification of the Living Mollusca. Melbourne, 189 pp.

Wall-Palmer D, Hegmann M, Goetze E, Peijnenburg KTCA (2019) Resolving species boundaries in the Atlanta brunnea species group (Gastropoda, Pterotracheoidea). ZooKeys 899: 59-84. https://doi.org/10.3897/zookeys.899.38892

Wu M, Chen ZY, Zhu XR (2019) Two new camaenid land snails (Eupulmonata) from Central China. ZooKeys 861: 129-144. https://doi.org/10.3897/zookeys.861.35430

Wu SP, Hwang CC, Lin YS (2008) Systematic revision of the arboreal snail Satsuma albida species complex (Mollusca: Camaenidae) with descriptions of fourteen new species from Taiwan. Zoological Journal of the Linnean Society 154: 437-493. https://doi.org/10.1111/ j.1096-3642.2008.00415.x

Yen TC (1939) Die chinesischen Land-und Süsswasser Gastropoden des Natur-Museums Senckenberg. Vittorio Klostermann, Frankfurt-am-Main. Abhandlungen der Senckenbergischen Naturforschenden Gesellschaft 444: 1-235.

Zilch A (1959-1960) Gastropoda. Teil 2: Euthyneura. Handbuch der Paläozoologie, Band 6. Berlin-Nikolassee, Gebrüder Borntraeger, 834 pp.

Zilch A (1964) Die Typen und Typoid des Natur-Museums Senckenberg, 29: Mollusca, Camaenidae (3). Archiv für Molluskenkunde 93: 243-262. 